\title{
Development of a framework and coding system for modifications and adaptations of evidence-based interventions
}

Shannon Wiltsey Stirman ${ }^{1,2,3^{*}}$, Christopher J Miller ${ }^{2,5}$, Katherine Toder ${ }^{4}$ and Amber Calloway ${ }^{6}$

\begin{abstract}
Background: Evidence-based interventions are frequently modified or adapted during the implementation process. Changes may be made to protocols to meet the needs of the target population or address differences between the context in which the intervention was originally designed and the one into which it is implemented [Addict Behav 2011, 36(6):630-635]. However, whether modification compromises or enhances the desired benefits of the intervention is not well understood. A challenge to understanding the impact of specific types of modifications is a lack of attention to characterizing the different types of changes that may occur. A system for classifying the types of modifications that are made when interventions and programs are implemented can facilitate efforts to understand the nature of modifications that are made in particular contexts as well as the impact of these modifications on outcomes of interest.

Methods: We developed a system for classifying modifications made to interventions and programs across a variety of fields and settings. We then coded 258 modifications identified in 32 published articles that described interventions implemented in routine care or community settings.

Results: We identified modifications made to the content of interventions, as well as to the context in which interventions are delivered. We identified 12 different types of content modifications, and our coding scheme also included ratings for the level at which these modifications were made (ranging from the individual patient level up to a hospital network or community). We identified five types of contextual modifications (changes to the format, setting, or patient population that do not in and of themselves alter the actual content of the intervention). We also developed codes to indicate who made the modifications and identified a smaller subset of modifications made to the ways that training or evaluations occur when evidence-based interventions are implemented. Rater agreement analyses indicated that the coding scheme can be used to reliably classify modifications described in research articles without overly burdensome training.
\end{abstract}

Conclusions: This coding system can complement research on fidelity and may advance research with the goal of understanding the impact of modifications made when evidence-based interventions are implemented. Such findings can further inform efforts to implement such interventions while preserving desired levels of program or intervention effectiveness.

Keywords: Implementation, Modification, Adaptation, Sustainability

\footnotetext{
* Correspondence: sws@bu.edu

'Women's Health Sciences Division, National Center for PTSD, Boston, MA,

USA

${ }^{2}$ VA Boston Healthcare System, Boston, MA, USA

Full list of author information is available at the end of the article
} 


\section{Background}

Evidence-based programs and interventions are frequently modified during the implementation process to address differences between the context in which the intervention was originally designed and tested, and the one into which it is ultimately implemented [1]. Descriptions of modifications in the research literature range broadly from slight changes in terminology or delivery in different languages, to removal of core components or integration with other interventions. Modifications can include adaptations, which are planned or purposeful changes to the design or delivery of an intervention, but they can also include unintentional deviations from the interventions as originally designed. That is, some modifications occur with the intention to retain fidelity to the fundamental elements or spirit of the intervention, whereas others may be unplanned changes made in reaction to a specific circumstance. Some may be relatively minor, while others might represent a significant change. Such variation in the nature of modifications can have very different implications for outcomes of interest. While some modifications might facilitate implementation and sustainability by improving the fit between the intervention and the target population or the context into which it is introduced, modifications may also erode treatment integrity. However, little research has been conducted to determine whether modifications of different natures compromise or enhance the desired benefits of interventions. Assessing fidelity, which comprises adherence to the intervention components, competence or skill with which the intervention is delivered, and differentiation from other treatments [2], by itself may fail to capture certain types of modifications (e.g., minor changes to terminology or language). While the recommended considerations for fidelity include unique and essential elements, necessary but not unique, acceptable but not necessary, and proscribed elements [3], most fidelity instruments do not contain an exhaustive listing of acceptable and proscribed behaviors. Thus, fidelity monitoring alone will not facilitate an understanding of whether different types of modifications are detrimental, non-detrimental or enhancements [4,5]. Without a better understanding of the nature and impact of modification, and the levels of fidelity necessary to promote desired outcomes, it is difficult to determine the best course of action with respect to the implementation of complex interventions in different contexts. This manuscript presents a comprehensive framework and model for classifying a broad range of modifications that may be made to evidence-based interventions. Such a framework, by quantifying the specific types and levels of modifications, can allow for more precise determination of the effects of such modifications on clinical or implementation outcomes of interest.
While the case has been made both for strict fidelity to interventions and for modifying interventions as necessary [4,6-10], few studies have examined the impact of modifications to treatments on health-related behaviors or outcomes. Among those studies, results have not been consistent. Levitt and colleagues compared outcomes of an intervention for post-traumatic stress disorder (PTSD) that included the option to use certain prescribed modifications, such as repeating or skipping modules, with clinical outcomes from a randomized controlled trial [11]. In this study, levels of fidelity to core intervention components remained high when the intervention was delivered with modifications, and PTSD symptom outcomes were comparable to those in a controlled clinical trial [11]. Galovski and colleagues also found positive outcomes when a highly specified set of adaptations were used in a different PTSD treatment [12]. Other studies have demonstrated similar or improved outcomes after modifications were made to fit the needs of the local audience and expand the target population beyond the original intervention. For example, an enhanced outcome was demonstrated after modifying a brief HIV risk-reduction video intervention to match presenter and participant ethnicity and sex [13]; effectiveness was also retained after modifying an HIV risk-reduction intervention to meet the needs of five different communities [14]. However, in other studies, modifications to enhance local acceptance appeared to compromise effectiveness. For example, Stanton and colleagues modified a sexual risk reduction intervention that had originally been designed for urban populations to address the preferences and needs of a more rural population, but found that the modified intervention was less effective than the original, unmodified version [15]. Similarly, in another study, cultural modifications that reduced dosage or eliminated core components of the Strengthening Families Program increased retention but reduced positive outcomes [16].

A challenge to a more complete understanding of the impact of specific types of modifications is a lack of attention to their classification. Some descriptions of intervention modifications and adaptations have been published (c.f. [17-19]), but there have been relatively few efforts to systematically categorize them. Researchers identified modifications made to evidence-based interventions such as substance use disorder treatments [1] and prevention programs [20] through interviews with facilitators in different settings. Others have described the process of adaptation (e.g., [21,22]). For example, Devieux and colleagues [23] described a process of operationalizing the adaptation process based on Bauman and colleagues' framework for adaptation [8], which includes efforts to retain the integrity of an intervention's causal/conceptual model. Other researchers 
[24-26] have also made recommendations regarding specific processes for adapting mental health interventions to address individual or population-level needs while preserving fidelity. Some work has been done to characterize and examine the impact of modifications made at the individual and population level. For example, Castro, Barrera and Martinez presented a program adaptation framework that described two basic forms of cultural adaptation: the modification of program content and modification of program delivery, and made distinctions between tailored and individualized interventions [27]. A description of personcentered interventions similarly differentiates between tailored, personalized, targeted and individualized interventions, all of which may actually lie on a continuum in terms of their complexity and comprehensiveness [27].

While these existing recommendations and models for adaptation provide critical guidance regarding the process of adaptation, particularly to improve the cultural relevance and individual-level 'fit' of interventions, adaptations may also occur to address provider needs or constraints in the intervention or program setting or healthcare system. Relatively little research has been conducted to empirically classify the nature of the full range of modifications made to interventions in routine care settings. Recognizing the benefits to identifying common types of modifications to evidence-based interventions, Hill and colleagues identified the types of modifications made for a single evidence-based prevention program in a statewide implementation [20]. Although they suggested that their study was a starting point in the development of a set of modification categories that generalize across interventions, the modifications that they identified were highly specific to the intervention that they examined. A more general and developed taxonomy can facilitate efforts to understand the nature of modifications that are made, whether by design or happenstance, as well as the impact of different types of modifications on implementation and health-related outcomes of interest. As a next step toward this understanding, it is necessary to identify the types of modifications that may occur when implementing interventions under a broad set of circumstances and in a variety of settings.

The purpose of this study was to develop a coding scheme to characterize modifications made to evidencebased interventions when they are implemented in contexts or with populations that differ from that in which they were originally developed or tested. Such a system can facilitate more systematic study of the types of modifications that are most commonly made across different contexts, populations and interventions. Additionally, it can provide a way to study the impact of different types of modifications on outcomes of interest. Hill and colleagues described a theory that a few types of modifications are likely to comprise the majority of all modifications that occur in practice [20]. Identifying what those modifications are, and what their impacts are for different interventions, can assist intervention developers and those who implement the interventions in determining and facilitating the range of modifications that are acceptable-and in preventing those that are not. We therefore sought to identify examples of a variety of modifications that practitioners, treatment developers, and other stakeholders made to a diverse set of interventions and programs. We intended this framework to apply in particular to three types of programs and interventions outlined by Scheirer [28]: those implemented by individual providers; programs requiring coordination among multiple staff; and new procedures aimed at targeting individual behaviors or behavioral health conditions.

\section{Method}

In developing the coding system, we searched the literature for articles published or in press before June, 2012, that assessed or described modifications to interventions implemented in routine service settings. We searched the following databases: Medline, ISI, PsycInfo, Academic Search Premier, Health Source, ERIC, Pubmed and Google Scholar, using the terms 'modif' or 'adapt' and 'evidence based treatment' or 'evidence-based intervention.' We also employed a snowballing strategy, in which we searched the reference sections of articles that we identified as well as theoretical papers on implementation that discussed modification and adaptation. Two authors (SWS, AC) reviewed abstracts and full text articles when necessary to determine their eligibility for this project and discussed one difference of opinion regarding inclusion with the rest of the study team. We included articles that provided sufficient detail about one or more modifications to facilitate coding. Modifications could be either adaptations (intentional, planned changes that typically included an effort to preserve fidelity) or changes that were made without premeditation during the delivery of the intervention. Articles were excluded if they assessed fidelity but did not describe modifications; if the interventions were developed, as opposed to being modified, for the purpose of the study or implementation project; or if they only provided recommendations for adaptation of an intervention without describing at least one specific modification that was made during the course of a research or implementation effort (Figure 1).

Once identified, articles were examined and segmented into discrete units for coding. One team member (SWS) segmented the articles into separate descriptions of modifications, and the remaining team members reviewed the entire articles and provided feedback regarding the accuracy of the segmentation process. Due to the dearth of previous work that has been done to actually classify a broad range of modifications, we used an analytic approach that 


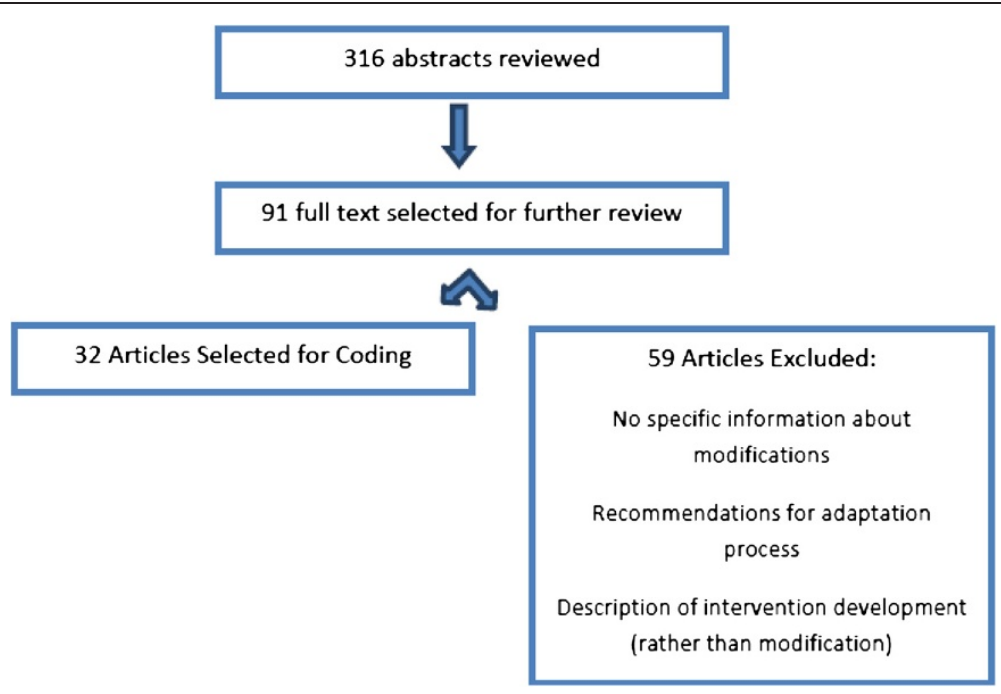

Figure 1 Article selection procedure and results.

was rooted in grounded theory $[29,30]$ to code the data from these articles. Using an iterative process, the study team examined the subset of identified segments that described modifications to identify emergent themes or categories. We then applied these themes to additional segments, allowing additional categories to emerge until theoretical saturation was achieved and a comprehensive coding scheme was developed. We specified characteristics of each category of modifications and ensured that the categories were mutually exclusive and exhaustive by combining or revising redundant codes. We also provided a draft of the framework and codebook to seven implementation researchers who were interested in the subject of modification and requested feedback. No additional codes or alternative classification structures were suggested, although some suggested combining some similar constructs into a single code and identified aspects of the codebook that could be clarified. After this feedback was incorporated, two team members (SWS, KT) then reexamined the article data, such that all segments identified in each article were coded with the finalized codebook. The two raters overlapped on $20 \%$ of the identified segments, and we computed Cohen's kappa coefficients [31] to determine interrater reliability for each rating category.

\section{Results}

A total of 32 articles were identified [9,11,13,15-18,20,32-51], which described 258 unique modifications (see Table 1). The types of intervention included preventive and health promotion interventions $(\mathrm{n}=15)$, mental or behavioral health $(n=13)$, behavioral medicine $(n=3)$, and a multidimensional complex care coordination intervention $(\mathrm{n}=1)$. Settings in which the interventions were delivered included hospitals and medical clinics, mental health clinics, substance abuse treatment programs, human service organizations, housing shelters, community organizations, employment settings, bars, and schools. Twenty-three articles provided author descriptions of modifications, four identified modifications through interviews with providers, two utilized observation or fidelity rating, and three based findings on a combination of observation and interviews.

\section{Classification of modifications}

Our coding process resulted in the identification of modifications to the context of program or intervention delivery, modifications to the intervention or program content itself, and modifications made during an implementation effort to training or evaluation processes. Furthermore, we included a code specifying who made the decision to make each modification. Figure 2 represents the coding system that emerged from this process, which is described in greater detail below. A comprehensive coding manual that includes decision rules and instructions regarding how to code each level is available by request from the first author. Contextual modifications include format, setting, channel of delivery and intervention recipients, and are about 'setting the stage' for an intervention to be delivered. Content modifications focus on the actual delivery of the intervention content. Training and evaluation modifications represent changes made 'behind the scenes' during an implementation effort. Although modifications to context and training/ evaluation codes were not always accompanied by substantial changes to the intervention content, we included them because it is possible that such changes could have an impact on fidelity, clinical outcomes, or the success of an implementation effort. Table 2 includes the frequency with which each modification occurred, along with rater agreement statistics. 
Table 1 Articles included in coding procedure

\begin{tabular}{|c|c|c|}
\hline Citation & Type of program & Source of modification data \\
\hline Aarons et al., [35] & Mental health & Behavioral observation \\
\hline Blasinsky, Goldman \& Unutzer, [46] & Collaborative care/mental health & Observation and interview \\
\hline Devieux et al., [23] & HIV/behavioral medicine & Author description \\
\hline Dushay et al., [41] & HIV Prevention & Author description \\
\hline Hasson, Blomberg \& Duner, [44] & Complex care & Observation and interview \\
\hline Hill, Maucione \& Hood, [20] & Substance abuse prevention & Clinician interview \\
\hline Hinton et al., [32] & Mental health & Author description \\
\hline Holliday et al., [51] & Health promotion & Observation and interview \\
\hline Kalichman et al., [13] & HIV Prevention & Author description \\
\hline Kaysen et al., [17] & Mental health & Author description \\
\hline Kelly et al., [9] & HIV Prevention & Author description \\
\hline Kennedy et al., [14] & HIV Prevention & Author description \\
\hline Kumpfer, Smith \& Bellamy, [16] & Prevention & Author description \\
\hline Leerlooijer et al., [19] & Sexual risk prevention & Author description \\
\hline Levitt et al., [11] & Mental health & Author description \\
\hline Lundgren et al., [1] & Substance abuse & Clinician interview \\
\hline Lyon et al., [38] & Mental health & Author description \\
\hline Malow et al., [39] & HIV Prevention & Author description \\
\hline McCabe et al., [33] & Mental health & Author description \\
\hline McIntyre, [38] & Mental health & Author description \\
\hline Melde, Esbensen \& Tusinski, [47] & Prevention & Behavioral observation \\
\hline Miller, [42] & HIV Prevention & Author description \\
\hline Nastasi et al., [21] & Sexual risk prevention & Author description \\
\hline Noonan et al., [49] & Sexual violence prevention & Clinician interview \\
\hline Owczarzak \& Dickson-Gomez, [37] & HIV Prevention & Clinician interview \\
\hline Remien et al., [48] & HIV/behavioral medicine & Author description \\
\hline Salerno et al., [18] & Mental health & Author description \\
\hline Stanton et al., [15] & Sexual risk prevention & Author description \\
\hline Tortolero et al., [22] & Sexual risk prevention & Author description \\
\hline Webster-Stratton \& Herman, [50] & Mental health & Author description \\
\hline Webster-Stratton \& Reid, [34] & Mental health & Author description \\
\hline Williams \& Williams, [45] & Behavioral medicine & Author description \\
\hline
\end{tabular}

\section{By whom was the decision to modify made?}

This code indicates the individual or group of individuals who made the decision regarding whether or how to modify the intervention. Cohen's kappa for this code was 0.80 , indicating substantial agreement.

1. Provider, practitioner, or facilitator: The individual who delivers the intervention made the modification.

2. Team/multiple providers: A group of providers modified the treatment (e.g., either an intervention that requires multiple providers is modified by those providers, or a unit of providers decide together to deliver a program or intervention in a different way).
3. Administrator or supervisor: The individual responsible for oversight of an individual provider, team, unit, organization or system decided how to modify the intervention or program.

4. Researcher: A researcher determined how to modify a program or intervention for the purposes of research (e.g., to study the impact of a particular adaptation or set of adaptations).

5. Purveyor or intervention developer: The individual who developed the intervention or an (often external) individual with expertise in the intervention who was tasked with supporting the implementation determined how to adapt or modify 


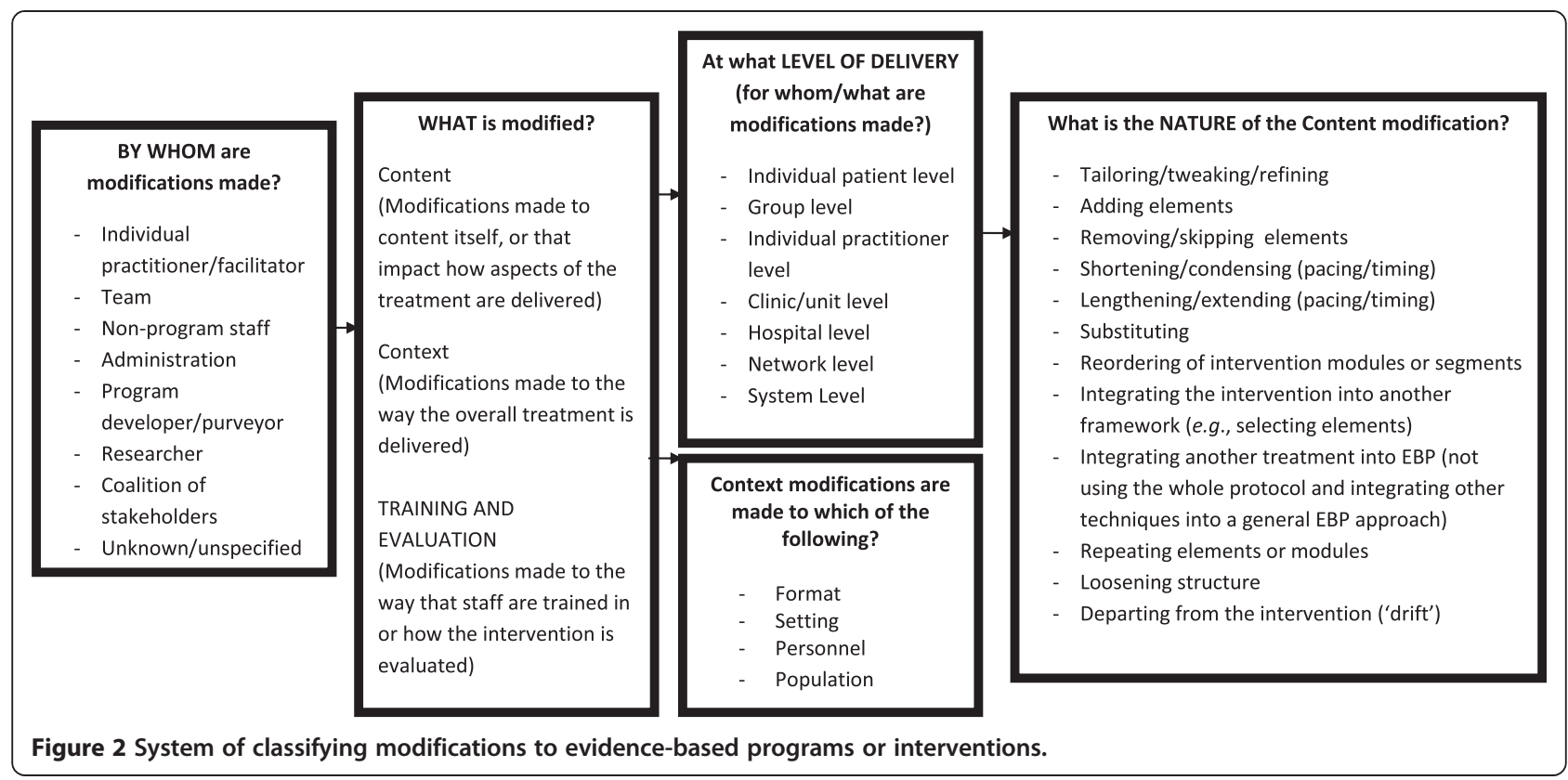

the treatment. If the purveyor and researcher are the same individual, the coding decision is made based on whether the modification is for research or implementation purposes.

6. Coalition of Stakeholders: A group of stakeholders actively participated in the decision-making regarding the types of modifications that are made to an intervention. If the purveyor or researchers used focus groups, interviews, or other means of gathering input to guide their decisions regarding modifications, this code was NOT used, unless stakeholders also directly participated in the process of using that information to adapt the intervention.

\section{Contextual modifications}

Similar to Castro and colleagues' description of differing forms of delivery [27], contextual modifications were defined as changes made to delivery of the same program content, but with modifications to the format or channel, the setting or location in which the overall intervention is delivered, or the personnel who deliver the intervention. We also include in this category the population to which an intervention is delivered. Modifications were only coded as contextual if an intervention was specifically designed for a particular context or population and then applied elsewhere or delivered in a different format than originally designed. Modifications were considered to be contextual if one of the elements described below was changed, whether or not alterations to the content of the intervention were made. When content-level changes were also made, they were coded separately. A total of 41 contextual modifications $(16 \%$ of the total sample of modifications) were described in the sample of articles. The subset of segments that was double-coded for reliability purposes indicated perfect agreement for the presence of contextual modifications.

1. Format: Changes are made to the format or channel of treatment delivery (e.g., a treatment originally designed to be used one-on-one that is now delivered in a group format).

2. Setting: The intervention is being delivered in a different setting or location (e.g., a treatment originally designed to be used in a mental health clinic setting that is now delivered in primary care).

3. Personnel: The intervention is being delivered by personnel with different characteristics (e.g., a treatment originally designed to be administered by a mental health professional is now delivered by clergy).

4. Population: An intervention that was specifically developed to target a particular population is being delivered to a different population than originally intended (e.g., an intervention developed for patients with Borderline Personality Disorder is now being delivered to individuals with Substance Dependence).

\section{Modifications to training and evaluation processes}

Changes made to the procedures for training personnel or evaluating the program are classified separately from content or contextual modifications, as they occur 'behind the scenes' and do not necessarily impact intervention content or the context of delivery. Examples include 
Table 2 Modifications and adaptations made to programs and interventions

\begin{tabular}{|c|c|c|c|}
\hline Decision maker (overall kappa $=0.80, n=261$ ) & Frequency & $\%$ of total modifications & \\
\hline Individual practitioner $^{\mathrm{a}}$ & 55 & 21 & \\
\hline Team or group of practitioners & 24 & 9 & \\
\hline Administrator & 2 & $<1$ & \\
\hline Researcher & 22 & 8 & \\
\hline Intervention developer or purveyor ${ }^{b}$ & 70 & 27 & \\
\hline Coalition of stakeholders ${ }^{c}$ & 76 & 29 & \\
\hline Unknown/insufficient information & 12 & 5 & \\
\hline Contextual modifications (overall kappa $=1.00, n=41$ ) & Frequency & $\%$ of contextual modifications & $\%$ of total modifications \\
\hline Format & 8 & 25 & 3 \\
\hline Setting & 6 & 19 & 2 \\
\hline Personnel & 10 & 3 & 4 \\
\hline Population & 8 & 25 & 3 \\
\hline Training and evaluation Processes & 9 & - & 3 \\
\hline Content modifications $(n=217)$ & Frequency & $\%$ of content modifications & $\%$ of total modifications \\
\hline \multicolumn{4}{|l|}{ Level (overall kappa $=0.79$ ) } \\
\hline Individual recipient & 14 & 6 & 5 \\
\hline Cohort & 14 & 6 & 5 \\
\hline Population $^{d}$ & 71 & 33 & 28 \\
\hline Provider/facilitator & 20 & 9 & 8 \\
\hline Unit $^{\mathrm{e}}$ & 38 & 18 & 15 \\
\hline Hospital/organization & 22 & 10 & 9 \\
\hline Network/community ${ }^{f}$ & 38 & 18 & 15 \\
\hline \multicolumn{4}{|l|}{ Nature of modification (overall kappa $=0.87$ ) } \\
\hline Tailoring/tweaking/refining ${ }^{f}$ & 73 & 34 & 28 \\
\hline Adding elements ${ }^{g}$ & 62 & 29 & 24 \\
\hline Removing elements ${ }^{9}$ & 33 & 15 & 13 \\
\hline Shortening/condensing ${ }^{\mathrm{h}}$ & 17 & 8 & 7 \\
\hline Lengthening/extending & 12 & 6 & 5 \\
\hline Substituting elements & 5 & 2 & 2 \\
\hline Re-ordering elements & 4 & 2 & 2 \\
\hline Integrating another approach into the intervention & 3 & 1 & 1 \\
\hline Integrating the intervention into another approach & 2 & 1 & 1 \\
\hline Departing from the intervention ("Drift") & 1 & $<1$ & $<1$ \\
\hline Loosening structure & 1 & $<1$ & $<1$ \\
\hline Repeating elements & 1 & $<1$ & $<1$ \\
\hline
\end{tabular}

Note: Three modifications that were identified in the articles did not contain sufficient detail to be rated.

${ }^{a}$ Raw agreement for this category was $100 \%$.

${ }^{\mathrm{b}}$ Raw agreement for this category was $82 \%$.

cRaw agreement for this category was $73 \%$.

${ }^{\mathrm{d}}$ Raw agreement for this category was $87 \%$.

${ }^{\mathrm{e}}$ Raw agreement for this category was $67 \%$.

fRaw agreement for this category was $91 \%$.

${ }^{9}$ Raw agreement for these categories was $83 \%$.

haw agreement for this category was $86 \%$.

expanding training from a single day to a three-day workshop, or making changes to the type of evaluation data or procedures for collecting evaluation data.

\section{Content modifications}

Content modifications are changes made to the intervention procedures, materials or delivery. They appear 
to occur at multiple levels and in differing contexts, ranging from changes made for an individual recipient to changes made uniformly across an entire network, community or system. Therefore, we included a code for both the level at which the modification was made (e.g., for a single patient vs. across the entire clinic), and the nature of the modification itself. A total of 217 content modifications ( $84 \%$ of all identified modifications) were described in the articles that were reviewed. Table 2 summarizes the frequency with which modification occurred at the following levels. Cohen's kappa for agreement on levels was 0.79 , indicating substantial agreement [52].

\section{Levels at which content modifications occur}

1. Individual recipient level: The intervention is modified for a particular recipient (e.g., simplifying language if a patient has cognitive impairment or if language barriers exist; changes to increase cultural relevance for an individual recipient).

2. Cohort level: The intervention is modified for individuals grouped within the intervention setting into a treatment group, a class, or other type of cohort (e.g., a specific psychotherapy group, grade or classroom).

3. Population level: The intervention is modified for application to a particular cultural, ethnic, clinical or social group (e.g., repetition of intervention components for all patients with cognitive impairments; development of culturally relevant vignettes to be used with all individuals of a particular ethnic identity).

4. Provider/facilitator level: Modifications are made by a clinician/facilitator for all of their participants (e.g., 'I never set an agenda when I do cognitive therapy').

5. Unit level: A modification is made by all of the facilitators in a unit (e.g., clinic/department/grade) within a larger organization (e.g., 'We can only do 60-minute intervention sessions instead of 90minute sessions in our clinic').

6. Hospital/Organization level: Modifications are made by an entire organization.

7. Network/Community level: Modifications are applied by an entire network or system of hospitals/ clinics/schools (e.g., a Veterans Affairs VISN; school district) or community.

\section{Types of content modifications}

We identified 12 different types of content modifications. Cohen's kappa for the nature of modifications was 0.87 , suggesting that rater agreement for these categories was in the 'almost perfect' range [52]. Regarding reliability for individual codes, raw agreement was at least $80 \%$ for each code that was applied more than 15 times in our dataset; less frequently-applied codes were not subjected to reliability analyses.

1. Tailoring/tweaking/refining: This code was assigned to any minor change to the intervention that leaves all of the major intervention principles and techniques intact while making the intervention more appropriate, applicable or acceptable (e.g., modifying language, creating slightly different versions of handouts or homework assignments, cultural adaptations).

2. Adding elements (intervention modules or activities): Additional materials or activities are inserted that are consistent with the fundamentals of the intervention (e.g., adding role play exercises to a unit on assertiveness in a substance abuse prevention intervention).

3. Removing elements (removing/skipping intervention modules or components): Particular elements of the intervention are not included (e.g., leaving out a demonstration on condom use in an HIV prevention intervention for adolescents).

4. Shortening/condensing (pacing/timing): A shorter amount of time than prescribed is used to complete the intervention or intervention sessions (e.g., shorter spacing between sessions, or shortening sessions, offering fewer sessions, or going through particular modules or concepts more quickly without skipping material).

5. Lengthening/extending (pacing/timing): A longer amount of time than prescribed by the manual/ protocol is spent to complete intervention or intervention sessions (e.g., greater spacing between sessions, longer sessions, more sessions, or spending more time on one or more modules/activities or concepts).

6. Substituting elements: A module or activity is replaced with something that is different in substance (e.g., replacing a module on condoms with one on abstinence in an HIV prevention program).

7. Re-ordering elements: Modules/activities or concepts are completed in a different order from what is recommended in the manual/protocol. This code would not be applied if the protocol allows flexibility in the order in which specific modules or interventions occur.

8. Integrating another approach into the intervention: The intervention of interest is used as the starting point, but aspects of different therapeutic approaches or interventions are also used (e.g., integrating an 'empty chair' exercise into a 'CBT for Depression' treatment protocol). 
9. Integrating the intervention into another approach: Another intervention is used as the starting point, but elements of the intervention of interest are introduced (e.g., integrating motivational enhancement strategies into a weight loss intervention protocol).

10.Repeating elements: One or more modules, sessions, or activities that are normally prescribed or conducted once during a protocol are used more than once.

11.Loosening structure: Elements intended to structure intervention sessions do not occur as prescribed in the manual/protocol (e.g., the 'check-in' at the beginning of a group intervention is less formally structured; clinician does not follow an agenda that was established at the beginning of the session).

12.Departing from the intervention ('drift'): The intervention is not used in a particular situation or the intervention is stopped, whether this stoppage was for part of a session or a decision to discontinue the intervention altogether (e.g., 'this client was so upset that I just spent the rest of today's session letting him talk about it instead of addressing his health behaviors').

\section{Discussion}

This study represents an effort to systematically characterize the types of modifications that are made to interventions when they are implemented in real world settings. On a high level, two of the major categories of coding mapped onto Castro and colleagues' distinction between modifications of program content, and modification of the form of delivery (e.g., location of delivery, delivery person, or channel of delivery) [27]. In a sample of studies described in peer-reviewed articles, which represent a variety of interventions and contexts, we found that contextual modifications were occasionally reported, but that content modifications were reported much more frequently. Tailoring the intervention to address language, cultural differences, literacy, or situational constraints was the most commonly identified content modification, followed by the addition or removal of elements and changes to the length or pacing of the intervention.

Other modifications identified in our coding process, such as drift and loosening of structure, occurred relatively rarely within the articles that we reviewed. This low frequency in the current sample is not surprising, as such behaviors are unlikely to occur in a planned manner, and may be less likely to be emphasized when describing an evidence-based intervention in a peerreviewed article. Furthermore, relatively few of the articles that were sampled employed the type of observation or stakeholder interviews through which such behaviors may be identified. While drift might also be considered a discontinuation of the intervention entirely or a lack of fidelity rather than a modification, it also seems important to capture it in a system designed to classify deviations from and modifications to a protocol in order to better measure its impact on outcomes of interest. For example, the impact of the option to occasionally or strategically drift on clinician or client satisfaction may be important to explore, in addition to the impact of drift on clinical effectiveness.

In contrast to the findings in Hill and colleagues' study [20], most of the articles that we found in our search process described modifications that were made proactively in recognition of key differences between the implementation setting and the original intervention. In another report, we describe findings that emerged when we applied this framework to interview data from a sample of community-based mental health service providers who were trained in an EBP [53]. Several of the lowerfrequency modifications identified in the current study were endorsed much more frequently in that study, suggesting that modifications made proactively may differ from those made once implementation is underway. Thus, at this stage of development, we determined that it is important to represent a more exhaustive set of possible modifications in the classification system.

As the discussion above indicates, some modifications may signify decreases in fidelity, while others may be consistent with the design of the intervention. The tension between modification and fidelity is a critical issue in implementation science $[4,54,55]$. Many recognize that modifications will occur throughout the course of an implementation effort, but the type and extent of modifications that can occur without compromising effectiveness or degrading fidelity to an unacceptable degree has not been sufficiently explored. In theory, it is possible to make some types of modifications without compromising effectiveness or removing the key elements of an intervention. However, for some interventions, the core elements have not yet been determined empirically, and very little is known about the impact of behaviors such as integrating other interventions or selectively implementing particular aspects of a treatment. Fidelity measures that emphasize competence or the spirit of an intervention over adherence may not adequately capture some potentially important types of modification, and those that emphasize adherence may not capture modifications such as tailoring. Thus, when observation or reliable self-report is possible, the use of a fidelity measure along with this modification framework can guide decisions regarding the extent to which a particular modification represents a departure from core elements of an intervention. Used alone or as a complement to fidelity measures, this measure may also be useful in 
determining whether particular elements can be removed, re-ordered, integrated or substituted without compromising effectiveness.

Despite the breadth of the coding system we developed, interrater agreement for the subset of independentlycoded articles was quite high, reaching standards of 'substantial agreement' and 'almost perfect' agreement for the level and nature of modifications, respectively [52]. Within our research group, this level of reliability was achieved after a brief series of hour-long weekly coding meetings, suggesting that our coding scheme can be used to reliably classify modifications described in research articles without overly burdensome training.

We note several potential limitations to the study and framework. First, our search process was not intended to identify every article that described modifications to evidence-based interventions, particularly if adaptation or modification was not a major topic addressed in the article. Instead, we sought to identify articles describing modifications that occurred across a variety of different interventions and contexts and to achieve theoretical saturation. In the development of the coding system, we did in fact reach a point at which additional modifications were not identified, and the implementation experts who reviewed our coding system also did not identify any new concepts. Thus, it is unlikely that additional articles would have resulted in significant additions or changes to the system.

In our development of this framework, we made a number of decisions regarding codes and levels of coding that should be included. We considered including codes for planned vs. unplanned modifications, major vs. minor modifications (or degree of modification), codes for changes to the entire intervention vs. changes to specific components, and codes for reasons for modifications. We wished to minimize the number of levels of coding in order to allow the coding scheme to be used in quantitative analyses. Thus, we did not include the above constructs, or constructs such as dosage or intensity, which are frequently included in frameworks and measures for assessing fidelity [56]. Additionally, we intend the framework to be used for multiple types of data sources, including observation, interviews and descriptions, and we considered how easily some codes might be applied to information derived from each source. Some data sources, such as observations, might not allow coders to discern reasons for modification or make distinctions between planned and unplanned modifications, and thus we limited the framework to characterizations of modifications themselves rather than how or why they were made. However, sometimes, codes in the existing coding scheme implied additional information such as reasons for modifying. For example, the numerous findings regarding tailoring interventions for specific populations indicate that adaptations to address differences in culture, language or literacy were common. Aarons and colleagues offer a distinction of consumerdriven, provider-driven, and organization-driven adaptations that might be useful for researchers who wish to include additional information regarding how or why particular changes were made [35]. While major and minor modifications may be easier to distinguish by consulting the intervention's manual, we also decided against including a code for this distinction. Some interventions have not empirically established which particular processes are critical, and we hope that this framework might ultimately allow an empirical exploration of which modifications should be considered major (e.g., having a significant impact on outcomes of interest) for specific interventions. Furthermore, our effort to develop an exhaustive set of codes meant that some of the types of modifications, or individuals who made the modifications, appeared at fairly low frequencies in our sample, and thus, their reliability and utility require further study. As it is applied to different interventions or sources of data, additional assessment of reliability and further refinement to the coding system may be warranted.

An additional limitation to the current study is that our ability to confidently rate modifications was impacted by the quality of the descriptions provided in the articles that we reviewed. At times, it was necessary to make some assumptions about how things were actually modified, or the level at which the modifications occurred. The level of detail available in records, clinical notes, or other qualitative data that may be utilized to investigate modifications may similarly impact future investigations. We attempted to address this limitation by making decision rules about the level of detail and clarity required to assign codes and by documenting these rules in detail in our coding manual. The level of rater agreement that we achieved suggests that our process was reasonably successful, despite occasional ambiguities in the descriptions. In future efforts to utilize this system, two strategies can minimize the likelihood that insufficient data are available to assign codes. Whenever possible, observation by raters knowledgeable about the intervention and its core components should be used to identify modifications. This may be especially important in differentiating minor modifications (which might be coded as 'tailoring/tweaking/refining') from more intensive modifications (which, for example, might be coded as 'removing elements'); ultimately, making these distinctions requires a thorough knowledge of the intervention itself. When interviews are conducted in lieu of observation or in addition to review of existing records, we recommend asking very specific follow-up questions regarding modifications that are made. Familiarity with both the intervention and the coding system when 
interviewing can increase the likelihood that sufficient information is obtained to make an appropriate judgment. Despite these measures, interrater reliability may vary across different data sources, although additional work by our research group suggests that reliability remains high when the coding scheme is applied to interview data [53]. We are currently examining reliability when the coding scheme is used for observation using audio recordings of psychotherapy sessions as well, and we recommend that when using this framework, researchers assess reliability.

We believe that the framework that we present can be used flexibly depending on the goals of the research and the type of data collection that occurs. For example, researchers may wish only to code exclusively for content or context-level modifications if they are interested in determining the impact of specific types of modifications on health outcomes. Similarly, the code for the decision maker may not be necessary if researchers are studying modifications made by one particular group or evaluating adaptations that were pre-specified by a single decisionmaker before implementation began. However, this code might be very informative if the researchers wish to understand the impact of the nature and process of modification on outcomes such as stakeholder engagement or fidelity to core program or intervention elements.

This coding system may be used to advance research that is designed with the goal of understanding the impact of changes made to interventions in particular contexts. Ultimately, such an understanding will require simultaneous use of this coding scheme and treatment outcome assessments, in order to help researchers and clinicians determine what specific types of modifications are most useful in increasing the effectiveness of interventions. Such an understanding will allow stakeholders to make more informed decisions about whether and how to modify the interventions when implementing them in contexts that differ from those in which they were originally developed and tested. Additionally, when used in the context of fidelity monitoring, this system can provide more useful information about what actually occurs when lower levels of adherence are identified, as well as the types of modifications that can occur within acceptable levels of fidelity. Baumann and colleagues suggested that there is a range of feasible fidelity, as well as a point of 'dramatic mutation', at which the intervention is no longer recognizable or effective [8]. This system of characterizing modifications may be useful in determining these ranges and boundaries with greater specificity. By understanding the types of modifications that can be made while keeping the intervention out of the range of dramatic mutation, stakeholders may ultimately find it easier to adapt interventions as needed while attending to an intervention's most critical components. Investigations of the impact of particular types of modifications on clinical outcomes can further inform efforts to implement evidence-based interventions while preserving desired levels of effectiveness. Finally, another potential area of investigation using this framework is on the impact of specific modifications on implementation outcomes such as adoption and sustainability. Additional knowledge about these critical issues in implementation science will yield important guidance for those wishing to advance the implementation of evidence-based programs and interventions.

\section{Competing interests}

The authors declare that they have no competing interests.

\section{Authors' contributions}

SWS conceptualized the study, contributed to the data collection and coding, and was the predominant contributor to this article. CM assisted with data analyses, assessment of reliability, interpretation of results, and contributed to the text of the manuscript. KT and AC assisted with literature searches, codebook development, and coded articles in the study. All authors did critical reading and modification of drafts and approved the final manuscript.

\section{Acknowledgments}

The preparation of this article was supported through funding from the National Institute of Mental Health (R00 MH 01800) [Dr. Stirman]. At the time that this study was being conducted, Dr. Stirman was a Fellow at the Implementation Research Institute (IRI), at the George Warren Brown School of Social Work, Washington University in St. Louis, which is funded through an award from the National Institute of Mental Health (R25 MH080916-01A2) and the Department of Veterans Affairs, Health Services Research \& Development Service, Quality Enhancement Research Initiative (QUERI). We wish to thank Jennifer Gamarra and Andrea DeVito for their assistance with the preparation of this manuscript. The content is solely the responsibility of the authors and does not necessarily represent the official views of the National Institute of Mental Health, the National Institutes of Health, or the Department of Veterans Affairs.

\section{Author details}

${ }^{1}$ Women's Health Sciences Division, National Center for PTSD, Boston, MA USA. ${ }^{2}$ VA Boston Healthcare System, Boston, MA, USA. ${ }^{3}$ Department of Psychiatry, Boston University, Boston, MA, USA. ${ }^{4}$ Department of Psychiatry, University of Pennsylvania, Philadelphia, PA, USA. ${ }^{5}$ VA Center for Organization, Leadership and Management Research, Boston, MA, USA. ${ }^{6}$ Department of Psychology, University of Massachusetts Boston, Boston, MA, USA.

Received: 18 September 2012 Accepted: 29 May 2013

Published: 10 June 2013

\section{References}

1. Lundgren $L$, et al: Modifications of evidence-based practices in community-based addiction treatment organizations: a qualitative research study. Addict Behav 2011, 36(6):630-635.

2. Schoenwald SK, et al: Toward the effective and efficient measurement of implementation fidelity. Adm Policy Ment Health 2011, 38(1):32-43.

3. Waltz J, et al: Testing the Integrity of a Psychotherapy Protocol: Assessment of Adherence and Competence. J Consult Clin Psychol 1993, 61(4):620-630.

4. Stirman SW, et al: The sustainability of new programs and interventions: A review of the empirical literature and recommendations for future research. Implementation Sci 2012, 7:17.

5. Zvoch K: Treatment fidelity in multisite evaluation. Am J Evaluation 2009, 30(1):44-61.

6. Dusenbury $L$, et al: $A$ review of research on fidelity of implementation: implications for drug abuse prevention in school settings. Health Educ Res 2003, 18(2):237-256.

7. Schoenwald SK GA, Chapman JE, Frazier SL, Sheidow AJ, Southam-Gerow MA: Toward the effective and efficient measurement of implementation fidelity. Administration Policy Mental Health and Mental Health Serv Res 2011, 38(1):32-43 
8. Bauman $\sqcup$, Stein REK, Ireys HT: Reinventing fidelity: the transfer of social technology among settings. Am J Community Psychol 1991, 19(4):619-639.

9. Kelly JA, et al: Transfer of research-based HIV prevention interventions to community service providers: fidelity and adaptation. AIDS EduC Prev 2000, 12:87-98.

10. Elliot DSMS: Issues in disseminating and replicating effective prevention programs. Prev Sci 2004, 5(1):47-53.

11. Levitt JT, et al: The flexible application of a manualized treatment for PTSD symptoms and functional impairment related to the $9 / 11$ World Trade Center attack. Behav Res Ther 2007, 45(7):1419.

12. Galovski TE, et al: Manualized therapy for PTSD: Flexing the structure of cognitive processing therapy. J Consult Clin Psychol 2012, 80(6):968-981.

13. Kalichman SC, et al: Culturally tailored HIV-AIDS risk-reduction messages targeted to African-American urban women:impact on risk sensitization and risk reduction. J Consult Clin Psychol 1993, 61(2):291-295.

14. Kennedy MG, et al: The effect of tailoring a model HIV prevention program for local adolescent target audiences. AIDS Educ Prev 2000, 12(3):225-238.

15. Stanton $B$, et al: The complex business of adapting effective interventions to new populations: an urban to rural transfer. J Adolesc Health 2005, 37(163):17-26.

16. Kumpfer KLAR, Smith P, Bellamy N: Cultural sensitivity and adaptation in family-based prevention interventions. Prev Sci 2002, 3(3):241-246.

17. Kaysen D, et al: Adaptation of cognitive processing therapy for treatment of torture victims: Experience in Kurdistan, Iraq. Psychological Trauma 2013, 52(2):184-192.

18. Salerno A, et al: Wellness self-management: an adaptation of the illness management and recovery program in New York State. Psychiatr Serv 2011, 62(5):456-458

19. Leerlooijer JN, et al: The world starts with me: using intervention mapping for the systematic adaptation and transfer of school based sexuality education from Uganda to Indonesia. TBM 2011, 1(2):331-340.

20. Hill LG, Maucione $K$, Hood BK: A focused approach to assessing program fidelity. Prev Sci 2007, 8:25-34.

21. Nastasi BK, et al: The participatory intervention model: a framework for conceptualizing and promoting intervention acceptability. School Psychology Quarterly 2000, 15(2):207-232.

22. Tortolero SR, et al: Using intervention mapping to adapt an effective HIV, sexually transmitted disease, and pregnancy prevention program for high-risk minority youth. Health Promot Pract 2005, 6(3):286-298.

23. Devieux JG, et al: Cultural adaptation in translational research: field experiences. J Urban Health 2005, 82(2 Suppl 3):iii82-iii91.

24. Goldstein NE, et al: Guidelines for adapting manualized interventions for new target populations: a step-wise approach using anger management as a model. Clin Psychology: Science and Practice 2012, 19(4):385-401.

25. Lau AS: Making the case for selective and directed cultural adaptations of evidence-based treatments: examples from parent training. Clin Psychology: Science \& Practice 2006, 13(4):295-310.

26. Aarons $\mathrm{G}$, et al: Dynamic adaptation process to implement an evidencebased child maltreatment intervention. Implementation Science 2012, 7(32).

27. Castro FG, Barrera M Jr, Martinez CR Jr: The cultural adaptation of prevention interventions: Resolving tensions between fidelity and fit. Prev Sci 2004, 5(1):41-45.

28. Scheirer MA: Linking Sustainability Research to Intervention Types. Am J Public Health 2013, 103(4):e73-e80.

29. Glaser BG, Strauss AL: The Discovery of Grounded Theory: Strategies for Qualitative Research. Chicago, IL: Aldine Publishing Company; 1967.

30. Strauss A, Corbin J: Basics of qualitative research. Techniques and procedures for developing grounded theory; 1998.

31. Cohen J: Weighted kappa: Nominal scale agreement provision for scaled disagreement or partial credit. Psychol Bull 1968, 70(4):213-220.

32. Hinton DEPT, Tran M, Safren SA, Otto MW, Pollack MH: CBT for Vietnamese refugees with treatment-resistant PTSD and panic attacks: a pilot study. J Trauma Stress 2004, 17(5):429-433.

33. McCabe KMYM, Garland AF, Lau AS, Chavez G: The GANA program: a tailoring approach to adapting parent child interaction therapy for Mexican Americans. Education \& Treatment of Children 2005, 28(2):111-129.

34. Webster-Stratton C, Reid M: Adapting the Incredible Years Child Dinosaur Social, Emotionalm and problem Solving Intervention to Address Comorbid Diagnoses. J Child Serv 2008, 3(3):17-30.

35. Aarons $\mathrm{G}$, et al: Adaptation happens: a qualitative case study of implementation of the incredible years evidence-based parent training program in a residential substance abuse treatment program.

$J$ Child Serv. in press.
36. McIntyre LL: Adapting Webster-Stratton's incredible years parent training for children with developmental delay: findings from a treatment group only study. J Intellect Disabil Res 2008, 52(12):1176-1192.

37. Owczarzak J, Dickson-Gomez J: Provider Perspectives on Evidence Based HIV Prevention Interventions: Barriers and Facilitators to Implementation. AIDS Patient Care STDS 2011, 25(3):171-179.

38. Lyon AR, et al: Easier Said Than Done: Intervention Sustainability in an Urban After School Program. AdministrationPolicy Mental Health and Mental Health Serv Res 2011, 38(6):504-517.

39. Malow RM, et al: Increasing access to preventative health care through cultural adaptation of effective HIV prevention interventions: a brief report from the HIV prevention in haitain youths study. ABNF J 2004, 132:127-132. November/December.

40. Devieux JG, Malow, Robert M, Rosenberg, Rhonda, Dyer, Janyce G: Context and common ground: cultural adaptation of an intervention for minority HIV Infected individuals. J Cult Divers 2004, 11(2):49-59.

41. Dushay RA, et al: Lowering HIV risk among ethnic minority drug users: comparing culturally targeted intervention to a standard intervention. Am J Drug Alcohol Abuse 2001, 27(3):501-524.

42. Miller RL: Adapting an evidence-based intervention: tales of the hustler project. AIDS Educ Prev 2003, 15(1):127-138.

43. Nastasi BK, et al: Participatory model of mental health programming: Lessons learned from work in a developing country. School Psychology Review 1998, 27(2):260-276.

44. Hasson H, Blomberg S, Duner A: Fidelity and moderating factors in complex interventions: a case study of a continuum of care program for frail elderly people in health and social care. Implementation Science 2012, 7(23).

45. Williams RB, Williams VP: Adaptation and implementation of an evidence based behavioral medicine program in diverse global settings: the Williams life skills experience. TBM 2011, 1(2):303-312.

46. Blasinsky M, Goldman HH, Unutzer J: Project IMPACT: A report on barriers and facilitators to sustainability. Administration Policy Mental Health and Mental Health Serv Res 2006, 33(6):718-729.

47. Melde C, Esbensen F-A, Tusinski K: Addressing Program Fidelity Using Onsite Observations and Program Provider Descriptions of Program Delivery. Eval Rev 2006, 30(6):714-740

48. Remien $\mathrm{RH}$, et al: Moving From Theory to Research to Practice: Implementing an Effective Dyadic Intervention to Improve Antiretroviral Adherence for Clinic Patients. Acquiring Immune Definciency Syndrome 2006, 43(1):S69-S78

49. Noonan R, et al: Adoption, Adaptation, and Fidelity of Implementation of Sexual Violence Prevention Programs. Health Promot Pract 2009, 10(1):59S-70S.

50. Webster-Stratton C, Reid MJ: Adapting The Incredible Years, an evidencebased parenting programme, for families involved in the child welfare system. J Child Serv 2010, 5(1):25-42.

51. Holliday J, et al: High Fidelity? How should we consider variations in the delivery of school based health promotion interventions? Health Educ $J$ 2009, 68(44).

52. Landis JR, Koch GG: The measurement of observer agreement for categorical data. Biometrics 1977, 33(1):159-174.

53. Stirman SW, et al: Modifications made to cognitive therapy by community mental health providers: Implications for effectiveness and sustainability. Psychiatr Serv 2013 (in press).

54. Shediac-Rizkallah MC, Bone LR: Planning for the sustainability of communitybased health programs: conceptual frameworks and future directions for research, practice and policy. Health Educ Res 1998, 13(1):87-108.

55. Scheirer MA, Dearing JW: An agenda for research on the sustainability of public health programs. Am J Public Health 2011, 101(11):2059-2067.

56. Bishop DC, et al: Measuring Fidelity and Adaptation: Reliability of a Instrument for School-Based Prevention Programs. Evaluation \& The Health Professions; 2013.

doi:10.1186/1748-5908-8-65

Cite this article as: Stirman et al.: Development of a framework and coding system for modifications and adaptations of evidence-based interventions. Implementation Science 2013 8:65. 